BRX-TH 415

\title{
Accelerated Detectors and Temperature in (Anti) de Sitter Spaces
}

\author{
S. Deser and Orit Levin
}

Physics Department, Brandeis University, Waltham, Massachusetts 02254, USA

\begin{abstract}
We show, in complete accord with the usual Rindler picture, that detectors with constant acceleration $a$ in de Sitter (dS) and Anti de Sitter (AdS) spaces with cosmological constants $\Lambda$ measure temperatures $2 \pi T=\left(\Lambda / 3+a^{2}\right)^{1 / 2} \equiv a_{5}$, the detector "5-acceleration" in the embedding flat 5 -space. For dS, this recovers a known result; in AdS, where $\Lambda$ is negative, the temperature is well defined down to the critical value $a_{5}=0$, again in accord with the underlying kinematics. The existence of a thermal spectrum is also demonstrated for a variety of candidate wave functions in AdS backgrounds.
\end{abstract}

A comoving detector in dS space is well known [1] to be in an effective thermal bath with temperature $2 \pi T=(\Lambda / 3)^{1 / 2}$ where $\Lambda$ is the (positive) cosmological constant, defined by $R_{\mu \nu \alpha \beta}=\frac{1}{2}\left(g_{\mu \alpha} g_{\nu \beta}-g_{\mu \beta} g_{\nu \alpha}\right) \Lambda$. This result was recently extended [2] to detectors with constant 4-acceleration $a$, whose temperature becomes $2 \pi T=\left(\Lambda / 3+a^{2}\right)^{1 / 2}$. Our main purpose here is to investigate the extent to which temperature is applicable in AdS geometry, despite its hyperbolicity problems, but we will for unity provide parallel treatments of both dS and AdS spaces, stressing in particular the usefulness of the embedding flat 5 -spaces, in which the detector's 5 -acceleration is precisely $\left(\Lambda / 3+a^{2}\right)^{1 / 2}$, for understanding the desired observer motions. We will of course recover the above dS result; in AdS we find the same formula, as might be expected on formal continuation grounds. However, the physics is quite different since there is a critical value $a^{2}=-\Lambda / 3$ of the acceleration below which the detector seems to measure imaginary temperature; the resolution is of course that its motion becomes spacelike. That these simple kinematical considerations indeed lead to the correct physics will be verified 
by the usual calculation of detector-quantum field interaction to exhibit a thermal distribution. For AdS, we will consider various boundary conditions that define states and show that they agree.

Our spaces are represented as hyperplanes in flat embedding $D=5$ spaces according to

$$
d s^{2}=\eta_{A B} d z^{A} d z^{B} \quad \eta_{A B} z^{A} z^{B}=\mp R^{2}
$$

$A, B=0 \ldots 4, \eta_{A B}=\operatorname{diag}(1,-1,-1,-1, \mp 1)$ for $\mathrm{dS} /$ AdS respectively and $R^{2} \equiv 3|\Lambda|$. Now consider detectors moving according to $z^{2}=z^{3}=0$ $z^{4}=Z=$ const; their 5 -space motions are

$$
\left(z^{1}\right)^{2}-\left(z^{0}\right)^{2}= \pm\left(R^{2}-Z^{2}\right) \equiv a_{5}^{-2}
$$

which resemble the hyperbolic trajectories

$$
x^{2}-t^{2}=a^{-2}
$$

of accelerated detectors in Minkowski space. There, such detectors measure temperature $2 \pi T=a$, so we would expect our observer (2) to see one as well, with

$$
2 \pi T=\left( \pm\left(R^{2}-Z^{2}\right)\right)^{-1 / 2} .
$$

These dS/AdS sign correlations agree with the formal fact that the spaces are related by a change of real to imaginary $R$ and $z^{4}$, but of course this kinematic argument has to be confirmed for both spaces, e.g., by exhibiting suitable Planck distributions for quantum fields in these backgrounds, and we must also express and understand these motions in the physical 4-space.

Consider first $\mathrm{dS}$, expressed in the standard coordinates covering the whole manifold

$$
d s^{2}=d t^{2}-R^{2} \cosh ^{2} t / R\left[d \chi^{2}+\sin ^{2} \chi\left(d \theta^{2}+\sin ^{2} \theta d \phi^{2}\right)\right],
$$

which are related to the $z^{A}$ of $\mathrm{SO}(1,4)$ according to

$$
\begin{aligned}
& z^{0}=R \sinh t / R, \quad z^{4}=R \cos \chi \cosh t / R, \quad z^{1}=R \sin \chi \cos \theta \cosh t / R \\
& z^{2}=R \sin \chi \sin \theta \cos \phi \cosh t / R \quad, \quad z^{3}=R \sin \chi \sin \theta \sin \phi \cosh t / R .
\end{aligned}
$$


For a radially moving detector, we may always (by the symmetry) choose $\theta=0, \phi=$ const or $z^{2}=z^{3}=0$. We further set $z^{4}=Z$, a constant or since $z^{1}$ and $z^{4}$ are on the same footing, another set of trajectories with the same acceleration is given by $z^{1}=Z=$ const. The two corresponding sets of (timelike) trajectories are illustrated in Fig 1. Because $|\cos \chi| \leq 1$, this means that $Z^{2} \leq R^{2} \cosh ^{2} t / R$, hence trajectories with $|Z|<R$ are timelike, $|Z|>R$ spacelike, and $|Z|=R$ null; we consider only $|Z|<R$ Next, let us evaluate the magnitude $a^{2}=-g_{\beta \gamma} a^{\beta} a^{\gamma}$ of the 4 -acceleration $D^{2} x^{\mu} / D s^{2}$. A straightforward calculation yields the constant value

$$
a^{2} R^{2}=Z^{2} /\left(R^{2}-Z^{2}\right) .
$$

Inserting $Z^{2}=a^{2} R^{4}\left(1+R^{2} a^{2}\right)^{-1}$ into (田), or using the relation $a_{5}^{2}=R^{-2}+a^{2}$ between the 5 - and 4 -accelerations (valid for every radial trajectory), yields the result of [2]

$$
2 \pi T=\left(R^{-2}+a^{2}\right)^{1 / 2} .
$$

The temperature found in (8) is also independent of the particular coordinates chosen. Thus it is the effective "Unruh acceleration" $a_{5}$ of the detector in the embedding flat 5 -space that determines the temperature.

The fundamental justification for the applicability of temperature is of course to be found in the form taken by quantum fields on the dS background. The Wightman function for conformally coupled massless scalar field has been shown [3] (see also [1]) to be identical to that in flat space, namely proportional to the square of the invariant 5-distance between the two points on the detector trajectory there. Hence an "Unruh effect" calculation has to give the same result as in flat space but now in terms of the 5-acceleration relevant in the embedding space. Thus the QFT justification of our result is immediate from this point of view. Equally convincing, of course, is the derivation of [2] based on the KMS interpretation of quantized fields [4] in dS.

We turn now to AdS, parametrized according to

$$
d s^{2}=R^{2}(\cos \rho)^{-2}\left[d \tau^{2}-d \rho^{2}-\sin ^{2} \rho\left(d \theta^{2}+\sin ^{2} \theta d \phi^{2}\right)\right]
$$

using

$$
\begin{aligned}
& z^{0}=R \sin \tau / \cos \rho \quad z^{4}=R \cos \tau / \cos \rho \quad z^{1}=R \tan \rho \cos \theta \\
& z^{2}=R \tan \rho \sin \theta \cos \phi \quad z^{3}=R \tan \rho \sin \theta \sin \phi ;
\end{aligned}
$$


the ranges $0 \leq \rho<\pi / 2,0 \leq \theta \leq \pi, 0 \leq \phi<2 \pi$ cover the whole manifold. We consider, as before, trajectories with $\theta=0, \phi=$ const, so that $z^{2}=z^{3}=0$, and the effective interval reduces to

$$
d s^{2}=R^{2}(\cos \rho)^{-2}\left(d \tau^{2}-d \rho^{2}\right) .
$$

A motion with $z^{1}$ or $\rho=$ const experiences the constant 4 -acceleration

$$
a^{2} R^{2}=\sin ^{2} \rho
$$

which is maximized at spatial infinity, $\rho=\pi / 2$, and vanishes at $\rho=0$. However, lines of constant $\rho$ are now realized as the circles $\left(z^{0}\right)^{2}+\left(z^{4}\right)^{2}=$ const in the embedding 5 -space; these no longer correspond to the hyperbolic trajectories a la Minkowski, and do not immediately suggest any thermal properties; we shall see below that they correspond to $T=0$. In contrast, we focus on the timelike lines $z^{4}=Z$ with $|Z|>R(|Z|<R$ are spacelike) that do follow hyperbolic trajectories,

$$
\left(z^{1}\right)^{2}-\left(z^{0}\right)^{2}=Z^{2}-R^{2}=a_{5}^{-2}
$$

with constant 4-acceleration

$$
a^{2} R^{2}=Z^{2}\left(\left(Z^{2}-R^{2}\right)\right)^{-1}
$$

Here $a$ ranges from $R^{-1}$ for $|Z|=\infty$ to infinity as $|Z|$ decreases from $\infty$ to $R$. Figure 2 exhibits the constant $z^{4}$ lines for AdS. The line $Z^{2}=\infty$ is $\rho=\pi / 2$, while the null lines $\tau= \pm \rho+n \pi$ are the $Z^{2}=R^{2}$ horizons: no detector can cross them. [We could have chosen an equivalent set of observers by setting $z^{0}$ rather than $z^{4}$ constant. These are translated by $\pi / 2$ in the $\tau$ direction with respect to the $z^{4}=Z$ choice; the horizons lines are likewise moved by $\pi / 2$.] We therefore expect, from the Minkowski-Rindler analogy in the flat embedding space description of detector motion, the AdS detectors on the $z^{4}=Z$ paths to measure the temperature

$$
2 \pi T=\left(-R^{2}+Z^{2}\right)^{-1 / 2}=\left(-R^{-2}+a^{2}\right)^{+1 / 2}
$$

As in $\mathrm{dS}$, the relation $a_{5}^{2}=-R^{-2}+a^{2}$ actually holds for every radial trajectory, but of course $T$ is only defined for constant accelerations. Since physical 
detectors cannot travel on the spacelike 4-orbits with $Z^{2}<R^{2}$, we need not worry about imaginary $T$.

Having motivated the existence and the form of the AdS temperature formula, we must now attempt to derive it. We use the Unruh approach -the excitation of a detector by a quantum field in the AdS background- thereby determining the temperature by the (lowest order) transition rate obtained from the Wightman function. Unlike the dS case, however there is an a priori problem: the lack of global hyperbolicity of AdS. We have nothing to add to this question, and will simply take over the results of [5] for defining a massless scalar field in a "causal" way, using the equivalent of box quantization. There is an ambiguity in defining the wave function $\Phi$, depending on the assumed boundary conditions, in terms of the wave function $\Phi_{E}$ of the static Einstein universe (into which AdS may be conformally mapped). The choices discussed in [5] are given respectively by $\Phi_{A}=(\cos \rho / R) \Phi_{E}$ ("transparent" boundary conditions), and two "reflective" conditions characterized by choosing $\Phi_{B}, \Phi_{C}$ such that they respectively obey the "wall" conditions $\partial \Phi_{E} / \partial \rho \rightarrow 0$ or $\Phi_{E} \rightarrow 0$ at $\rho \rightarrow \pi / 2$. For each of these 3 choices, we can compute the Wightman function to obtain

$$
\begin{gathered}
\left.8 \pi^{2} R^{2} W_{n}\left(x, x^{\prime}\right)\right|_{\theta=\theta^{\prime} \phi=\phi^{\prime}}=X\left[1+\varepsilon_{n}(1+2 X)^{-1}\right] \\
X=\frac{\cos \rho \cos \rho^{\prime}}{\left[\cos \left(\tau-\tau^{\prime}-i \epsilon\right)-\cos \left(\rho-\rho^{\prime}\right)\right.} \quad\left(\varepsilon_{A}, \varepsilon_{B}, \varepsilon_{C}\right)=(0,-1,1)
\end{gathered}
$$

for cases A,B,C respectively. For $\rho=$ const trajectories, $\tau=s \cos \rho / R$ where $s$ is proper time, so $W$ depends only on proper time differences. The corresponding transition rate is then of the form

$$
\Gamma_{n}=A \int_{-\infty}^{\infty} d\left(s-s^{\prime}\right) \exp \left[-i \Delta E\left(s-s^{\prime}\right)\right] W_{n}\left(s-s^{\prime}\right)
$$

$A$ is a constant depending on the detector-scalar field interaction and $\Delta E$ the energy difference between detector energy levels. We can immediately check that the integral vanishes for $\rho=$ const trajectories (all the pole in $W_{n}$ are in the upper half-plane) so that temperature vanishes, in agreement with the non-hyperbolic nature of these paths noted above. For the $z^{4}=Z$ trajectories we can solve for the proper time and express everything in terms of it and the acceleration to find that

$$
X=a_{5}^{2} R^{2}\left[1-\cosh a_{5}\left(s-s^{\prime}\right)-i \epsilon\right]^{-1} .
$$


Hence $X$ is a function of $\left(s-s^{\prime}\right)$ only and vanishes as $\left(s-s^{\prime}\right) \rightarrow \infty$, so we may use the adiabatic transtion rate (17). The contribution of $X$ to $\Gamma$ is

$$
\Gamma_{A}=\frac{A \Delta E}{2 \pi}\left[\exp \left(2 \pi \Delta E / a_{5}\right)-1\right]^{-1},
$$

the same expression as for the constant acceleration detector in Minkowski space [1], so that our original expectation is fulfilled. The other two cases differ from A by extra terms, which after some arithmetic are found to be

$$
\Gamma_{B, C}=\Gamma_{A} \pm \frac{A}{2 \pi} \frac{a_{5} \sin \left[b \Delta E / a_{5}\right]}{\sinh b}\left[\exp \left(2 \pi \Delta E / a_{5}\right)-1\right]^{-1}
$$

where $b \equiv 1+2 a_{5}^{2} R^{2} \geq 1$. For fixed $a_{5}$, the limit as $R \rightarrow \infty$ (flat space) of these extra terms reassuringly vanishes. More generally, we argue that the dominant term here is still the Planck denominator, so that the notion of temperature persists, as does its magnitude also for the $\mathrm{B}$ and $\mathrm{C}$ boundary conditions.

In summary, we have established a Rindler-like kinematical basis for associating temperature with constant accelerated motions in AdS (as in dS) geometries, their effective accelerations in the embedding flat 5-space being the relevant parameter. In AdS, this picture also accounted for the existence of a threshhold value of the 4-acceleration above which temperature is welldefined. These considerations were confirmed by explicit calculations of the correlators of a quantum field in AdS, whose result was independent of the different boundary conditions permitted by the AdS causality ambiguities, and led to the standard Unruh Planckian temperature distribution.

SD is happy to thank the authors of [2] for stimulating conversations. This work was supported by NSF grant PHY-9315811, OL by the Fishbach Foundation.

\section{References}

[1] Birrell N.D. and Davies P.C.W. 1982 Quantum Fields in Curved Space (Cambridge: Cambridge University Press) 
[2] Narnhofer H., Peter I. and Thirring W. 1996 Int. J. Mod. Phys. B 10 1507

[3] Tagirov E.A. 1973 Ann. Phys. 76561

[4] Bros J. and Moschella U. 1996 Rev. Math. Phys. 8327

[5] Avis S.J., Isham C.J. and Storey D. 1978 Phys. Rev. D 183565 
Fig 1 Penrose diagram of dS space. Solid lines represent $z^{4}= \pm|Z|$, broken lines the two possible sets with $z^{1}=Z$. Dotted lines give the null horizons of the trajectory sets to which they are the asymptotes.

Fig 2 AdS space. Solid lines represent different sets of $z^{4}=Z$ trajectories: $Z$ negative for $-3 \pi / 2<\tau<-\pi / 2, Z$ positive for $-\pi / 2<\tau<\pi / 2$, etc, alternating in each $\pi$-interval. The dotted lines are the corresponding null horizons. 


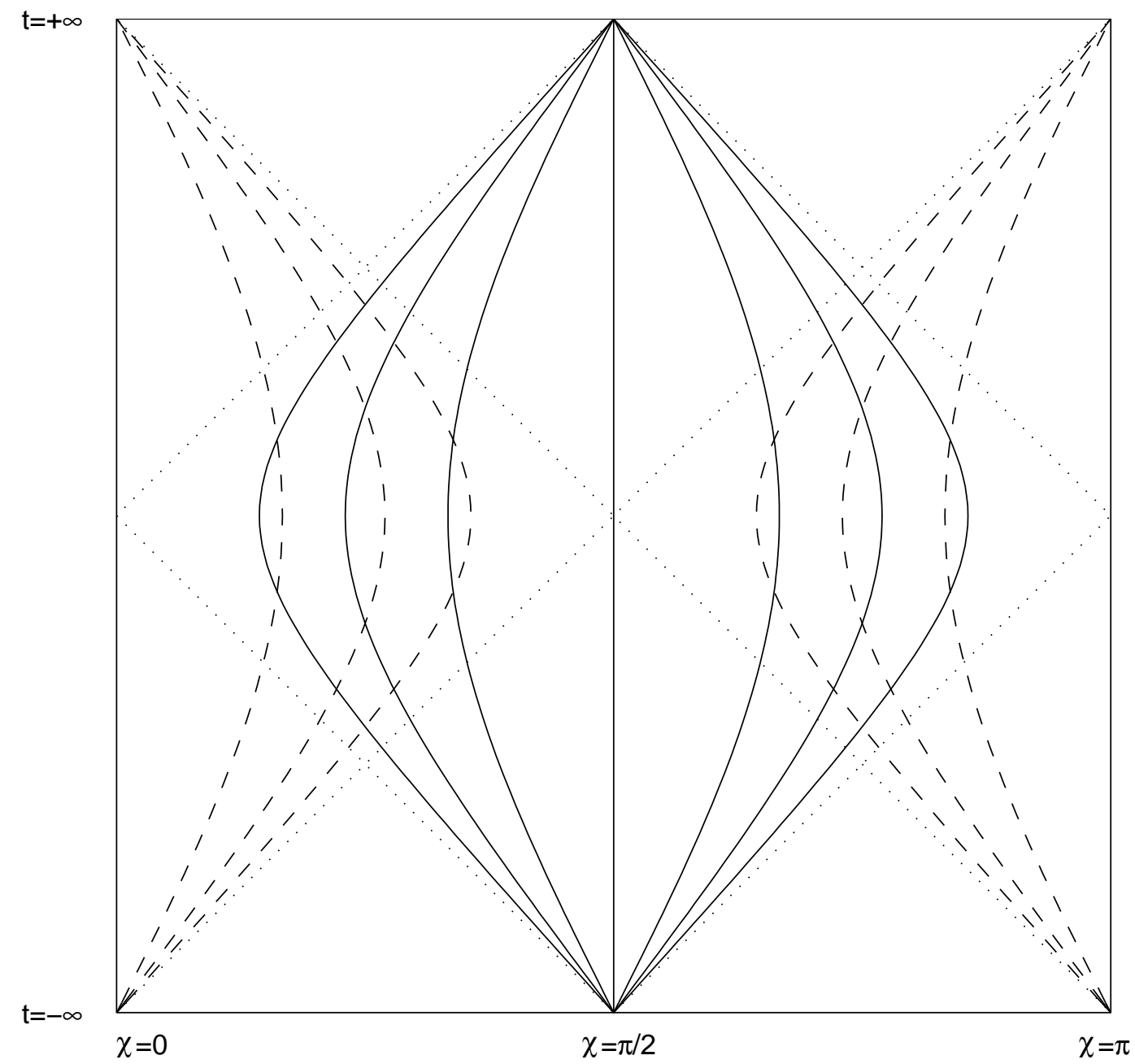




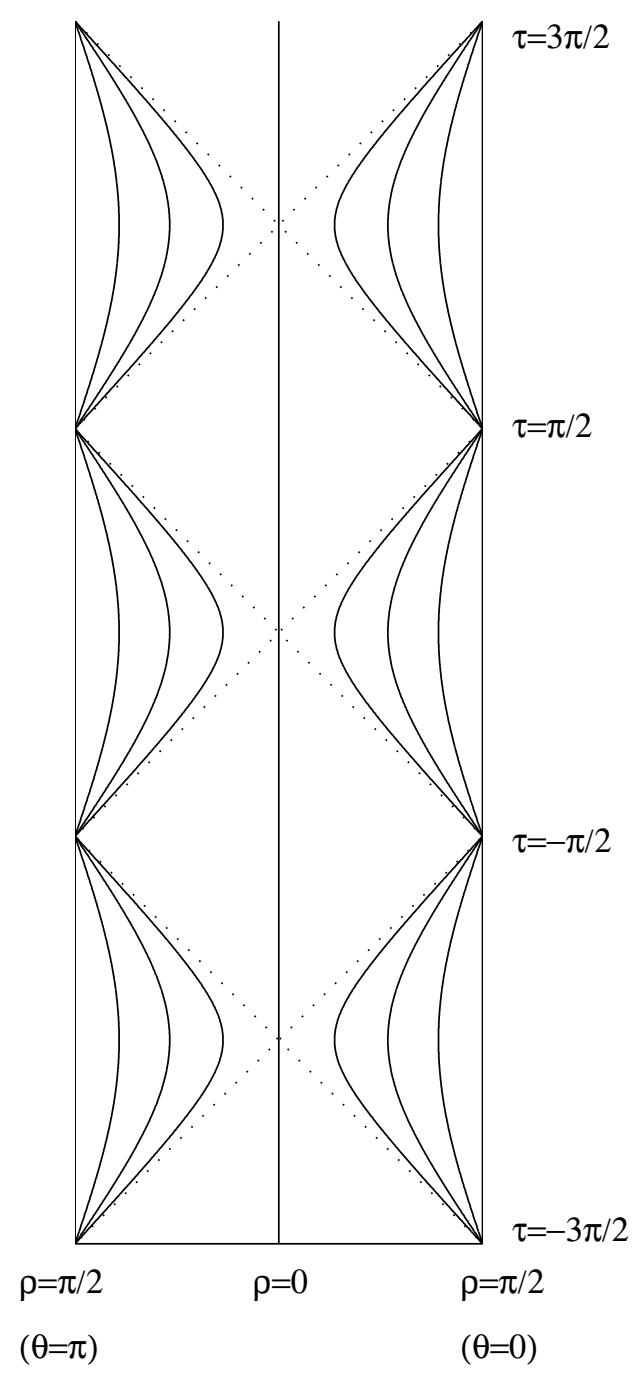

